\title{
A Proposal of PSO Particles' Initialization for Costly Unconstrained Optimization Problems: ORTHOinit
}

\author{
Matteo Diez ${ }^{1}$, Andrea Serani ${ }^{1,2}$, Cecilia Leotardi ${ }^{1}$, Emilio F. Campana ${ }^{1}$, \\ Daniele Peri ${ }^{3}$, Umberto Iemma ${ }^{2}$, Giovanni Fasano ${ }^{4}$, and Silvio Giove ${ }^{5}$ \\ 1 Natl. Research Council-Marine Tech. Research Inst. (CNR-INSEAN), Italy \\ $\{$ (matteo.diez, emiliofortunato. campana\}@cnr.it, c.leotardi@insean.it \\ 2 University 'Roma Tre', Department of Engineering, Italy \\ \{andrea.serani, umberto. iemma\}@uniroma3.it \\ 3 Natl. Research Council-Ist. Applicazioni del Calcolo 'M. Picone', (CNR-IAC), Italy \\ d.peri@iac.cnr.it \\ 4 University Ca'Foscari of Venice, Department of Management, Italy \\ fasano@unive.it \\ ${ }^{5}$ University Ca'Foscari of Venice, Department of Economics, Italy \\ sgiove@unive.it
}

\begin{abstract}
A proposal for particles' initialization in PSO is presented and discussed, with focus on costly global unconstrained optimization problems. The standard PSO iteration is reformulated such that the trajectories of the particles are studied in an extended space, combining particles' position and speed. To the aim of exploring effectively and efficiently the optimization search space since the early iterations, the particles are initialized using sets of orthogonal vectors in the extended space (orthogonal initialization, ORTHOinit). Theoretical derivation and application to a simulation-based optimization problem in ship design are presented, showing the potential benefits of the current approach.
\end{abstract}

Keywords: Global Optimization, Derivative-free Optimization, Deterministic PSO, Particles' Initial Position and Velocity.

\section{Introduction}

In this paper we consider the solution of the global unconstrained optimization problem

$$
\min _{x \in \mathbb{R}^{n}} f(x),
$$

where $f: \mathbb{R}^{n} \rightarrow \mathbb{R}$ is continuous and possibly nondifferentiable. In particular, we aim at detecting a global minimum $x^{*}$ of (1), satisfying $f\left(x^{*}\right) \leq f(x)$, for any $x \in \mathbb{R}^{n}$. Of course we assume that (1) admits solution, which may be guaranteed under mild assumptions on $f(x)$ (e.g., $f(x)$ is coercive with $\lim _{\|x\| \rightarrow \infty} f(x)=$ $+\infty)$. Furthermore, we also assume that the function $f(x)$ is computationally expensive, which possibly discourages the use of asymptotically convergent methods (i.e., iterative methods that only eventually ensure convergence properties to stationary points). 
PSO is an iterative method for global optimization, based on updating a population of points (namely particles). Preliminary numerical tests performed in [1, for 60 standard problems, suggest that the initial choice of particles' position/velocity may affect significantly the performance of PSO, giving motivation for further investigation of apparently basic and well stated issues for PSO (such as the choice of the initial particles' position/velocity).

Herein, we study the trajectories of particles in an extended space, so that analytical indications will be available in order to suggest the setting of initial particles position and velocity. The current approach starts from considering the results obtained in 223/4/5]6, though our partial conclusions in Sections 4/5]are, to the best of our knowledge, novel in the literature.

In Sections 2[3] we first recall the reformulation of PSO, detailed in [7] and [8], while the new proposal of this paper is in Sections 4 45 . Conclusions and future work are presented in Section 6 . In the following, ' $I$ ' indicates the identity matrix and ' $e_{i}$ ' is the $i$-th unit vector. The Euclidean norm is simply indicated by $\|\cdot\|$.

\section{A Reformulation of PSO Iteration}

Consider the following standard (and complete) iteration of PSO:

$$
\begin{cases}v_{j}^{k+1}=\chi\left[w^{k} v_{j}^{k}+c_{j}^{k} r_{j}^{k}\left(p_{j}^{k}-x_{j}^{k}\right)+c_{g}^{k} r_{g}^{k}\left(p_{g}^{k}-x_{j}^{k}\right)\right], & k \geq 0, \\ x_{j}^{k+1}=x_{j}^{k}+v_{j}^{k+1}, & k \geq 0,\end{cases}
$$

where $j=1, \ldots, P$ indicates the $j$-th particle, $P$ is finite, $v_{j}^{k}$ and $x_{j}^{k}$ are the velocity and the position of particle $j$ at step $k$, and the coefficients $\chi, w^{k}, c_{j}^{k}, r_{j}^{k}, c_{g}^{k}, r_{g}^{k}$ are bounded. Finally, $p_{j}^{k}$ and $p_{g}^{k}$ satisfy

$$
p_{j}^{k}=\underset{0 \leq h \leq k}{\operatorname{argmin}}\left\{f\left(x_{j}^{h}\right)\right\}, \quad j=1, \ldots, P, \quad p_{g}^{k} \underset{0 \leq h \leq k, j=1, \ldots, P}{\operatorname{argnin}}\left\{f\left(x_{j}^{h}\right)\right\} .
$$

We can also generalize (2) and the analysis in this paper by assuming that possibly the velocity $v_{j}^{k+1}$ depends on all the terms $p_{h}^{k}-x_{j}^{k}, h=1, \ldots, P$, obtaining the so called fully informed PSO (FIPS) 9]. This corresponds to allow a more general social contribution in PSO iteration. Notwithstanding the latter choice, we prefer to keep the notation as simple as possible, considering the recurrence (2) as is. Without loss of generality at present we focus on the $j$ th particle and omit the subscript in the recurrence (2), so that $p_{j}^{k}=p^{k}$ and $v_{j}^{k}=v^{k}$.

Assumption 1. We assume in (2) that $c_{j}^{k}=c, r_{j}^{k}=r$ for any $j=1, \ldots, P$, $c_{g}^{k}=\bar{c}, r_{g}^{k}=\bar{r}$ and $w^{k}=w$, for any $k \geq 0$.

Using the latter position the iteration (2) is equivalent to the dynamic, linear and stationary system 1

$$
X(k+1)=\left(\begin{array}{cc}
\chi w I & -\chi(c r+\bar{c} \bar{r}) I \\
\chi w I & {[1-\chi(c r+\bar{c} \bar{r})] I}
\end{array}\right) X(k)+\left(\begin{array}{c}
\chi\left(c r p^{k}+\bar{c} \bar{r} p_{g}^{k}\right) \\
\chi\left(c r p^{k}+\bar{c} \bar{r} p_{g}^{k}\right)
\end{array}\right),
$$

\footnotetext{
${ }^{1}$ See also 78 , whose terminology and symbols are simply reported in this brief section and in the next one. Then, in Section 4 we extend the latter results to our purposes.
} 
where

$$
X(k)=\left(\begin{array}{c}
v^{k} \\
x^{k}
\end{array}\right) \in \mathbb{R}^{2 n}, \quad k \geq 0 .
$$

The sequence $\{X(k)\}$ identifies a trajectory in the state space $\mathbb{R}^{2 n}$, and since (44) is a linear and stationary system, we may consider the free response $X_{L}(k)$ and the forced response $X_{F}(k)$ of the trajectory $\{X(k)\}$. Then, considering (4) we explicitly obtain, at step $k \geq 0, X(k)=X_{L}(k)+X_{F}(k)$, where

$$
X_{L}(k)=\Phi(k) X(0), \quad X_{F}(k)=\sum_{\tau=0}^{k-1} H(k-\tau) U(\tau),
$$

and, after some calculations

$$
\begin{gathered}
\Phi(k)=\left(\begin{array}{cc}
\chi w I & -\chi(c r+\bar{c} \bar{r}) I \\
\chi w I & {[1-\chi(c r+\bar{c} \bar{r})] I}
\end{array}\right), H(k-\tau)=\left(\begin{array}{cc}
\chi w I & -\chi(c r+\bar{c} \bar{r}) I \\
\chi w I & {[1-\chi(c r+\bar{c} \bar{r})] I}
\end{array}\right)^{k-\tau-1}, \quad(6) \\
U(\tau)=\left(\begin{array}{c}
\chi\left(c r p^{k}+\bar{c} \bar{r} p_{g}^{k}\right) \\
\chi\left(c r p^{k}+\bar{c} \bar{r} p_{g}^{k}\right)
\end{array}\right) .
\end{gathered}
$$

A remarkable observation from the latter formulae is that $X_{L}(k)$ in (5) uniquely depends on the initial point $X(0)$, and is not affected by the vector $p_{g}^{k}$. On the contrary, $X_{F}(k)$ in (5) is independent of $X(0)$, being strongly dependent on $p_{g}^{k}$. This implies that the quantities $X_{L}(k)$ and $X_{F}(k)$ can be separately computed.

\section{Structural Properties of Matrix $\Phi(k)$ and Computation of $X_{L}(k)$}

In order to simplify our analysis, provided that Assumption 1 holds, hereafter we consider the following position in (6)

$$
a=\chi w, \quad \omega=\chi(c r+\bar{c} \bar{r}) .
$$

Now, we first recall (see [7/24]) that in order to ensure necessary conditions which avoid divergence of the trajectories of particles, the relations

$$
0<|a|<1, \quad 0<\omega<2(a+1)
$$

must hold. Moreover, the only two eigenvalues $\lambda_{1}$ and $\lambda_{2}$ of $\Phi(1)$ coincide if and only if $\omega=(1 \pm \sqrt{a})^{2}$. Thus, if $\omega \neq(1 \pm \sqrt{a})^{2}$ then the results in [7] can be applied, so that $X_{L}(k)=[\Phi(1)]^{k} X(0)$ can be computed by simply introducing the eigenvalues $\lambda_{1}$ and $\lambda_{2}$ of $\Phi(1)$, yielding the formula

$$
[\Phi(1)]^{k} X(0)=\left[\begin{array}{c}
\gamma_{1}(k) v^{0}-\gamma_{2}(k) x^{0} \\
\gamma_{3}(k) v^{0}-\gamma_{4}(k) x^{0}
\end{array}\right],
$$

where

$$
\begin{array}{ll}
\gamma_{1}(k)=\frac{\lambda_{1}^{k}\left(a-\lambda_{2}\right)-\lambda_{2}^{k}\left(a-\lambda_{1}\right)}{\lambda_{1}-\lambda_{2}} & \gamma_{2}(k)=\frac{\omega\left(\lambda_{1}^{k}-\lambda_{2}^{k}\right)}{\lambda_{1}-\lambda_{2}} \\
\gamma_{3}(k)=\frac{\left(\lambda_{1}^{k}-\lambda_{2}^{k}\right)\left(a-\lambda_{1}\right)\left(a-\lambda_{2}\right)}{\omega\left(\lambda_{1}-\lambda_{2}\right)} & \gamma_{4}(k)=\frac{\lambda_{1}^{k}\left(a-\lambda_{1}\right)-\lambda_{2}^{k}\left(a-\lambda_{2}\right)}{\lambda_{1}-\lambda_{2}} .
\end{array}
$$




\section{A Novel Starting Point for Particles in PSO}

In this section we study a novel strategy to possibly improve the efficiency of PSO, based on the idea of widely exploring the search space in the early iterations, while maintaining the PSO iteration (2). As stated in the Introduction, our analysis seems more promising when each function evaluation is particularly expensive and time resources are scarce, so that a few iterations of PSO are allowed. We start our analysis using the reformulation in Section 2 in order to impose a novel condition for the choice of initial particles' position/velocity (namely the next relation (14)).

Consider two particles, namely particle $j$ and particle $h$, such that $1 \leq j \neq$ $h \leq P$; using the theory in Section 2 we can consider their trajectories in the space $\mathbb{R}^{2 n}$, so that their initial position and free response are respectively given by (see (100)

Particle j:

$$
X(0)^{(j)}=\left(\begin{array}{c}
v_{j}^{0} \\
x_{j}^{0}
\end{array}\right) \Rightarrow X_{L}(k)^{(j)}=[\Phi(1)]^{k} X(0)^{(j)}=\left[\begin{array}{c}
\xi_{1}(k)^{(j)} v_{j}^{0}-\xi_{2}(k)^{(j)} x_{j}^{0} \\
\xi_{3}(k)^{(j)} v_{j}^{0}-\xi_{4}(k)^{(j)} x_{j}^{0}
\end{array}\right],
$$

Particle h:

$$
X(0)^{(h)}=\left(\begin{array}{c}
v_{h}^{0} \\
x_{h}^{0}
\end{array}\right) \Rightarrow X_{L}(k)^{(h)}=[\Phi(1)]^{k} X(0)^{(h)}=\left[\begin{array}{l}
\xi_{1}(k)^{(h)} v_{h}^{0}-\xi_{2}(k)^{(h)} x_{h}^{0} \\
\xi_{3}(k)^{(h)} v_{h}^{0}-\xi_{4}(k)^{(h)} x_{h}^{0}
\end{array}\right],
$$

where $\xi_{i}(k)^{(h)}, i=1, \ldots, 4$ (similarly for $\left.\xi_{i}(k)^{(j)}\right)$ coincide with $\gamma_{i}(k), i=$ $1, \ldots, 4$, if $\omega \neq(1 \pm \sqrt{a})^{2}$.

Now, observe that at iteration $k$ the velocity $v^{k}$ of a particle may be regarded as a search direction from the current position $x^{k}$. Thus, we can be interested to find out conditions on the initial position of the particles, in order to possibly guarantee the orthogonality of particles' velocity at any iteration $k \geq 0$. The latter fact is expected to possibly favour a better exploration in $\mathbb{R}^{2 n}$. However, the latter condition is very tough to impose, without strongly modifying PSO iteration (2). Nonetheless, following the idea in Section 6 of [7, we can attempt for any $k$ to impose the orthogonality of the free responses $\left\{X_{L}(k)^{(j)}\right\}$. In particular, numerical efficiency is ensured by the fact that it is possible to set the initial position and velocity of $n$ particles, in such a way that the corresponding free responses $X_{L}(k)^{j_{1}}, \ldots, X_{L}(k)^{j_{n}}$ satisfy

$$
\left[X_{L}(k)^{j_{i}}\right]^{T}\left[X_{L}(k)^{j_{h}}\right]=0, \quad \forall j_{i}, j_{h} \in\left\{j_{1}, \ldots, j_{n}\right\}, \quad i \neq h .
$$

In order to generalize the latter idea we observe here that what really matters is the orthogonality of the search directions of the particles, and possibly not the orthogonality of the entire free responses. On this guideline, here we study the initial position and velocity of $2 n$ particles, so that for any $k$ the corresponding free responses $X_{L}(k)^{j_{1}}, \ldots, X_{L}(k)^{j_{2 n}}$ satisfy for any $1 \leq j \neq h \leq 2 n$ (see (11)(12))

$$
\left[\xi_{1}(k)^{(j)} v_{j}^{0}-\xi_{2}(k)^{(j)} x_{j}^{0}\right]^{T}\left[\xi_{1}(k)^{(h)} v_{h}^{0}-\xi_{2}(k)^{(h)} x_{h}^{0}\right]=0 .
$$


I.e., only the first $n$ entries of the free responses of particles $j$ and $h$, corresponding to the velocity, are orthogonal. After some computation, the latter relation is equivalent to the conditions

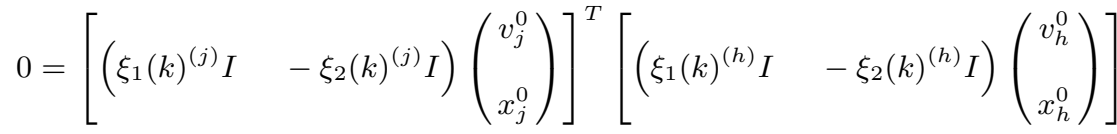

$$
\begin{aligned}
& =\left(\begin{array}{c}
v_{j}^{0} \\
x_{j}^{0}
\end{array}\right)^{T}\left[\begin{array}{cc}
\sigma_{1} I & \sigma_{2} I \\
\hat{\sigma}_{2} I & \sigma_{3} I
\end{array}\right]\left(\begin{array}{c}
v_{h}^{0} \\
x_{h}^{0}
\end{array}\right) \text {, }
\end{aligned}
$$

where $\sigma_{1}=\xi_{1}(k)^{(j)} \xi_{1}(k)^{(h)}, \sigma_{2}=-\xi_{1}(k)^{(j)} \xi_{2}(k)^{(h)}, \hat{\sigma}_{2}=-\xi_{2}(k)^{(j)} \xi_{1}(k)^{(h)}$, $\sigma_{3}=\xi_{2}(k)^{(j)} \xi_{2}(k)^{(h)}$.

Observe that setting the same parameters $\omega$ and $a$ in (8) for all the particles (i.e., for any $k \geq 0$ we have $\xi_{1}(k)^{(j)}=\xi_{1}(k)^{(h)}=\xi_{1}(k)$ and $\xi_{2}(k)^{(j)}=\xi_{2}(k)^{(h)}=$ $\left.\xi_{2}(k)\right)$ the matrix

$$
\Lambda=\left[\begin{array}{ll}
\sigma_{1} I & \sigma_{2} I \\
\hat{\sigma}_{2} I & \sigma_{3} I
\end{array}\right]=\left[\begin{array}{ll}
\sigma_{1} I & \sigma_{2} I \\
\sigma_{2} I & \sigma_{3} I
\end{array}\right]
$$

is symmetric and condition (14) indicates that the vectors

$$
\left(\begin{array}{c}
v_{j}^{0} \\
x_{j}^{0}
\end{array}\right), \quad\left(\begin{array}{c}
v_{h}^{0} \\
x_{h}^{0}
\end{array}\right) .
$$

must be mutually conjugate (see also 1011 for a reference). The first relevant property induced by the introduction of conjugacy is that conjugate vectors are linearly independent. This implies that in case the vectors (16) are mutually conjugate, then not only the velocities of the free responses of the particles are orthogonal (as stated in relation (13)), but the vectors (16) will be also sufficiently well scattered in $\mathbb{R}^{2 n}$. Now, note that if $z_{i}$ and $z_{j}$ are distinct eigenvectors of matrix $\Lambda$, respectively associated to the eigenvalues $\lambda_{i}$ and $\lambda_{j}$, then we simply have $z_{i}^{T} \Lambda z_{j}=z_{i}^{T}\left(\lambda_{j} z_{j}\right)=\lambda_{j} z_{i}^{T} z_{j}=0$, where the last equality follows from the fact that distinct eigenvectors of a symmetric matrix are orthogonal. Thus, the eigenvectors of a symmetric matrix are also mutually conjugate directions with respect to that matrix. As a consequence, in order to satisfy condition (14) it suffices to compute the eigenvectors of (15), and set the vectors in (16) as proportional to the latter eigenvectors. After some computation we have for the corresponding $2 n$ eigenvectors $u_{1}^{(i)}, u_{2}^{(i)}, i=1, \ldots, n$, of the matrix in (15) the simple expressions

$$
u_{1}^{(i)}=\left(\begin{array}{c}
-\frac{\sigma_{3}-\mu_{-}}{\sigma_{2}} e_{i} \\
e_{i}
\end{array}\right) \in \mathbb{R}^{2 n}, u_{2}^{(i)}=\left(\begin{array}{c}
-\frac{\sigma_{3}-\mu_{+}}{\sigma_{2}} e_{i} \\
e_{i}
\end{array}\right) \in \mathbb{R}^{2 n}, i=1, \ldots, n,
$$

where $\mu_{\mp}=\left[\left(\sigma_{1}+\sigma_{3}\right) \mp \sqrt{\left(\sigma_{1}+\sigma_{3}\right)^{2}-4\left(\sigma_{1} \sigma_{3}-\sigma_{2}^{2}\right)}\right] / 2$ are the eigenvalues of matrix $\Lambda$.

The last result implies that in order to satisfy the conditions (14), for any $1 \leq j \neq h \leq P \leq 2 n$, it suffices to set the initial particle position and velocity 
(respectively of the $i$-th and $(n+i)$-th particle) according with the following (ORTHOinit) initialization

$$
\begin{aligned}
\left(\begin{array}{c}
v_{i}^{0} \\
x_{i}^{0}
\end{array}\right) & =(-1)^{\alpha_{1 i}} \rho_{i}^{1} u_{1}^{(i)}, \quad \rho_{i}^{1} \in \mathbb{R} \backslash\{0\}, \alpha_{1 i} \in\{0,1\}, i=1, \ldots, n \\
\left(\begin{array}{c}
v_{n+i}^{0} \\
x_{n+i}^{0}
\end{array}\right) & =(-1)^{\alpha_{2 i}} \rho_{i}^{2} u_{2}^{(i)}, \quad \rho_{i}^{2} \in \mathbb{R} \backslash\{0\}, \alpha_{2 i} \in\{0,1\}, i=1, \ldots, n .
\end{aligned}
$$

Recalling that the choice of the coefficients $\rho_{i}^{1}, \rho_{i}^{2}, i=1, \ldots, n$, in (18)-(19) is arbitrary, we conclude that, in case in (8) $\omega \neq(1 \pm \sqrt{a})^{2}$, then

- when $P \leq 2 n$, the choice (18)-(19) of the particles position and velocity guarantees that the components of velocity of the free responses of the particles will be orthogonal at any iteration $k \geq 0$, provided that Assumption 1 holds (i.e., no randomness is used in PSO, as in DPSO [12]);

- in case $P>2 n$, the user can adopt the choice (18)-(19) of the particles position and velocity for $2 n$ particles, while setting the remaining $(P-2 n)$ particles arbitrarily.

\section{$5 \quad$ Numerical Results}

Numerical results for both test functions and a simulation-based design of a highspeed catamaran are performed using DPSO, setting the parameters according to Assumption 1 .

Numerical experiments are performed to assess the initialization (18)-(19) on the 60 test functions in [1, varying the initialization of the swarm. Three approaches are used. Specifically, in the first approach the swarm is initialized as shown in [1, using $4 n$ particles distributed (following a Hammersley sequence sampling, HSS) over the variables domain and its boundary. The second approach, following the guidelines in the previous sections, consists of using two orthogonal sets of $2 n$ particles each (ORTHOinit initialization). For the third approach, an orthogonal set of $2 n$ particles is added to the initialization set of the first approach. As shown in Figures 1 and 2, using two ORTHOinit sets of $2 n$ particles gives the best performance in terms of evaluation metric $\Delta$ (see [1]), for test functions with both $n<10$ and $n \geq 10$ design variables.

For the catamaran design optimization, the parent hull considered is that of the Delft catamaran, a concept ship used for experimental and numerical benchmarks. The optimization problem is taken from [13] and solved by means of stochastic radial-basis functions interpolation [14 of high-fidelity URANS simulations. Six design variables control global shape modifications, based on the Karhunen-Loève expansion of the shape modification vector [15. The objective is the reduction of the total resistance in calm water at Froude number equal to 0.5 . Figure 3 plots the decrease of the objective function in the first twenty DPSO iterations (early iterations), comparing the reference implementation given in 


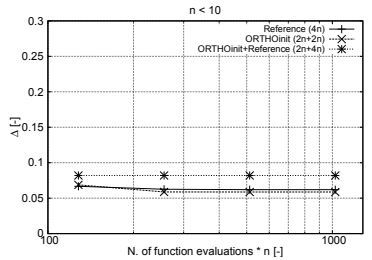

Fig. 1. Test funct., $n<10$

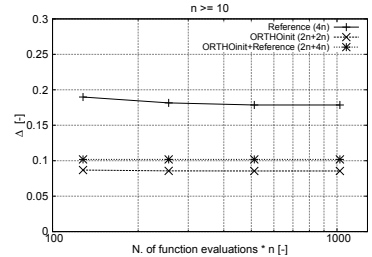

Fig. 2. Test funct., $n \geq 10$

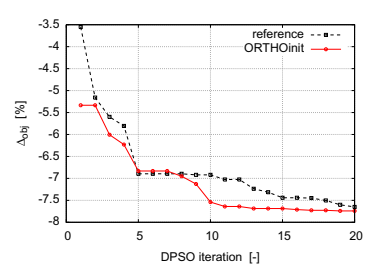

Fig. 3. Delft catamaran

[1, based on HSS initialization of particles over domain and boundary, with the method in this paper ( $4 n$ particles are used). ORTHOinit shows a faster progress than the reference implementation, confirming the effectiveness of the present method when a reduced number of iterations is allowed.

\section{Conclusions and Future Work}

With respect to 7 the theory above yields a guideline for the choice of $2 n$ (and not just $n$ ) particles' initial position/velocity. This was expected to provide a more powerful tool (as numerical results seem to confirm) for the exploration of the search space. Moreover, the above theory proposes a particles' initialization in PSO which is related to the space dimension $n$. Though no specific conclusion seems to be drawn by the latter observation, note that most of the exact derivative-free methods for smooth problems, as well as gradient-based methods for continuously differentiable functions, show some analogies. We are persuaded that in our framework an adaptive criterion might be advisable, in order to restart the position and velocity of the particles after a given number of iterations. The latter criterion can indeed monitor the norm $\left\|X_{L}(k)^{(j)}\right\|, j=1, \ldots, 2 n$ (see also Section 5 of [7]), of the free response of particles. When the latter quantity approaches zero, a restart would re-impose orthogonality among the free responses of the particles, using the theory in Section 4 .

Acknowledgements. The work of M. Diez is supported by the US Navy Office of Naval Research, NICOP Grant N62909-11-1-7011, under the administration of Dr. Ki-Han Kim and Dr. Woei-Min Lin. The work of A. Serani, C. Leotardi, G. Fasano and E.F. Campana is supported by the Italian Flagship Project RITMARE, coordinated by the Italian National Research Council and funded by the Italian Ministry of Education, within the National Research Program 2011-2013.

\section{References}

1. Serani, A., Diez, M., Leotardi, C., Peri, D., Fasano, G., Iemma, U., Campana, E.F.: On the use of synchronous and asynchronous single-objective deterministic Particle Swarm Optimization in ship design problems. In: Proceeding of OPT-i, International Conference on Engineering and Applied Sciences Optimization, Kos Island, Greece, June 4-6 (2014) 
2. Clerc, M., Kennedy, J.: The Particle Swarm - Explosion, Stability, and Convergence in a Multidimensional Complex Space. IEEE Transactions on Evolutionary Computation 6(1) (2002)

3. Ozcan, E., Mohan, C.K.: Particle Swarm Optimization: Surfing the Waves. In: Proceedings of the 1999 IEEE Congress on Evolutionary Comnputation, pp. 1939-1944. IEEE Service Center, Piscataway (1999)

4. Trelea, I.C.: The particle swarm optimization algorithm: convergence analysis and parameter selection. Information Processing Letters 85, 317-325 (2003)

5. Van den Berg, F., Engelbrecht, F.: A Study of Particle Swarm Optimization Particle Trajectories. Information Sciences Journal (2005)

6. Poli, R.: The Sampling Distribution of Particle Swarm Optimisers and their Stability, Technical Report CSM-465, University of Essex (2007)

7. Campana, E.F., Fasano, G., Pinto, A.: Dynamic analysis for the selection of parameters and initial population, in particle swarm optimization. Journal of Global Optimization 48, 347-397 (2010)

8. Campana, E.F., Diez, M., Fasano, G., Peri, D.: Initial particles position and parameters selection for PSO. In: Tan, Y., Shi, Y., Mo, H. (eds.) ICSI 2013, Part I. LNCS, vol. 7928, pp. 112-119. Springer, Heidelberg (2013)

9. Mendes, R., Kennedy, J., Neves, J.: The fully informed particle swarm: Simpler, maybe better. IEEE Transactions on Evolutionary Computation 8, 204-210 (2004)

10. Hestenes, M.R., Stiefel, E.: Methods of conjugate gradients for solving linear systems. Journal of Research of the National Bureau of Standards 49, 409-435 (1952)

11. Fasano, G., Roma, M.: Iterative Computation of Negative Curvature Directions in Large Scale Optimization. Computational Optimization and Applications 38, 81-104 (2007)

12. Campana, E.F., Liuzzi, G., Lucidi, S., Peri, D., Piccialli, V., Pinto, A.: New Global Optimization Methods for Ship Design Problems. Optimization and Engineering 10, 533-555 (2009)

13. Chen, X., Diez, M., Kandasamy, M., Zhang, Z., Campana, E.F., Stern, F.: Highfidelity global optimization of shape design by dimensionality reduction, metamodels and deterministic particle swarm. Engineering Optimization (in press, 2014), doi: 10.1080/0305215X.2014.895340

14. Volpi, S., Diez, M., Gaul, N.J., Song, H., Iemma, U., Choi, K.K., Campana, E.F., Stern, F.: Development and validation of a dynamic metamodel based on stochastic radial basis functions and uncertainty quantification. Structural Multidisciplinary Optimization (in press, 2014), doi: 10.1007/s00158-014-1128-5

15. Diez, M., He, W., Campana, E.F., Stern, F.: Uncertainty quantification of Delft catamaran resistance, sinkage and trim for variable Froude number and geometry using metamodels, quadrature and Karhunen-Loève expansion. Journal of Marine Science and Technology 19(2), 143-169 (2014), doi:10.1007/s00773-013-0235-0 